Second Meeting, 8th December 1905.

D. C. M'Irtosh, Esq., M.A., President, in the Chair.

\title{
A Problem of Lewis Carroll's, and the rational solutions of a Diophantine Cubic.
}

By C. Tweedie, M.A., B.Sc.

§1. In the Life and Letters of Lewis Carroll occurs the following extract from his Diary :-

“Dec. 19 (Sun). - Sat up last night till 4 a.m., over a tempting problem, sent me from New York, 'to find 3 equal rational-sided rt.-angled $\triangle$ 's.' I found two, whose sides are $20,21,29 ; 12,35,37$; but could not find three.' (v. page 343.)

The first object of this paper is to show how, starting from any given rational-sided right-angled triangle, we can certainly deduce other two of like area. A simple geometrical construction for a series (finite or infinite) of such triangles is also given.

$\$ 2$. Diophantine problems of this kind have always had a great fascination for mathematicians, and the most famous of them, known as Fermat's Last Theorem, $\left(x^{n}+y^{n}=z^{n}\right.$ has no rational solutions for $n>2$ ) still awaits a satisfactory solution.

When rational solutions of an equation with rational coefficients are in question, geometrical methods of investigation may often be successfully employed. Consider the equation $f(x, y, 1)=0$. Its solutions may be represented graphically in a plane space by means of a curve, and we have to determine "rational points" on this curve.

In the case of an equation of the first degree in $x$ and $y$ to every rational value of one coordinate corresponds a rational value of the other.

For a quadratic equation

$$
(a, b, c, f, g, h, \not x y 1)^{2}=0
$$

if one rationsl point $(\xi, \eta)$ exists, all the others may be found as follows. Take any "rational" line $y-\eta=m(x-\xi)$ through $(\xi, \eta)$ 
where $m$ has any rational value. Its second intersection with the conic represented by the quadratic equation is a rational point, and variation of $m$ will give all such points. Thus $(0,-1)$ is on the curve $x^{3}+y^{2}=1$, and therefore all other rational points are given by $2 m /\left(1+m^{2}\right),\left(1-m^{2}\right) /\left(1+m^{2}\right)$, where $m$ has any rational value. It follows that the sides of a rational-sided right-angled triangle are given by $2 \rho m ; \rho\left(1-m^{2}\right) ; \rho\left(1+m^{2}\right)$. For the rational solutions of the equation $\alpha^{2}+\beta^{2}=\gamma^{2}$ correspond to the rational solutions of

$$
(a / \gamma)^{2}+(\beta / \gamma)^{2}=1
$$

\$3. In the problem before us we have to find all rational solutions of the equation $\alpha^{2}+\beta^{2}=\gamma^{2}$, subject to the condition $\alpha \beta=2 \mathrm{~A}$ where $\mathrm{A}$ is a constant area; i.e., to find the rational solutions of

$$
\begin{aligned}
& x_{1}^{2}+y_{1}{ }^{2}=1 \\
& \gamma^{2} x_{2} y_{2}=2 \mathrm{~A} .
\end{aligned}
$$

subject to the condition

where $\gamma$ is a suitable rational quantity.

The rational solutions of (1) are given by

$$
2 m /\left(1+m^{2}\right),\left(1-m^{2}\right) /\left(1+m^{2}\right) .
$$

We have therefore to find all rational values of $m$ and $\gamma$ for which

$$
2 \gamma^{2} m\left(1-m^{2}\right)=2 \mathrm{~A}\left(1+m^{2}\right)^{2}
$$

Write $x$ for $m$ and $y$ for $\left(1+m^{2}\right) / \gamma$, when we have to determine the rational points on the curve

$$
x\left(1-x^{2}\right)=\mathrm{A} y^{2} .
$$

The two sides of the corresponding triangle are $2 x / y$ and $\left(1-x^{2}\right) / y$, and the hypotenuse is $\left(1+x^{2}\right) / y$.

\$. Description of the cubic curve.

Equation (3) represents a non-unicursal cubic, which, when $\boldsymbol{A}$ is positive, consists of an elliptic oval for $x$ between 0 and +1 , and an infinite serpentine branch between $x=-1$, and $x=-\infty$, both symmetrical with respect to the $x$-axis.

Differentiation with respect to $x$ gives the equations

$$
\begin{array}{ll}
2 A y y^{\prime} & =1-3 x^{2} \\
2 A y^{\prime 2}+2 A y y^{\prime \prime} & =-6 x
\end{array}
$$

There is therefore no real inflexion when $x$ is positive (A positive). The inflexions have their abscisse given by

$$
x^{2}=(3 \pm 2 \sqrt{ } 3) / 3
$$


and there are, as in other cubics, only three real inflexions given by

$$
x=-\sqrt{ }\{(3+2 \sqrt{ } 3) / 3\},
$$

and by the point of inflexion at infinity. It is a harmonic cubic, i.e., the four tangents from any point on it, touching the curve elsewhere, form a pencil of lines whose cross ratio is constant and equal to -1 . This may be seen by taking in particular the tangents from the inflexion at infinity which cut the $x$-axis at $(-1,0) ;(0,0) ;(1,0) ;(\infty, 0)$. As in all other cubics consisting of two branches, there is an "even" branch and an "odd" branch. Every line cuts the oval in two points real, coincident or imaginary, or not at all. No real tangents can be drawn from the oval to touch the cubic elsewhere. Also, it will appear presently that the four tangents from any point on the "odd" branch are real. Two of the tangents touch the oval, and two touch the serpentine elsewhere. (v. Schröter, Theorie der ebenen Kurven dritter Ordnung.) It follows naturally that all "tangentials" are points on the odd branch. It is besides clear that, since the oval is elliptic and does not possess any real inflexion, two real tangents can be drawn to it from any point "outside" it. Regarding cubics in general it will only be necessary to assume that if $A_{1} B_{1} C_{1} ; A_{2} B_{2} C_{2}$ are two chords of a cubic, and if $A_{1} A_{2}, B_{1} B_{2}, C_{1} C_{2}$ again cut the curve in $A_{3}, B_{3}, C_{3}$, then the latter three points are collinear. This theorem will be denoted as $I$. and quoted under the form

$$
\begin{array}{lll}
A_{1} & B_{1} & C_{1} \\
A_{2} & B_{2} & C_{2} \\
A_{d} & B_{3} & C_{3}
\end{array}
$$

\$5. Rational solutions of a cubic.

The solution of the general cubic equation was first given in its general form by Cauchy, Exercices de Math. cahier 4. (v. also Desboves Nouv. Annales, 1886.) Two methods are given. In geometrical language they are as follows:-(i) If a rational point on the cubic is known, its tangential-the remaining point of intersection of the tangent at the point with the cubic-furnishes a second rational point; and (ii) If two rational points on a cubic are known, the line joining them cuts the cubic again in a rational point. Neither method is perfect, and either is liable to exception. Thus (i) breaks down for the point $(0,1)$ on $x^{3}+y^{3}=1$, for this point is a point of inflexion. Similarly (ii) breaks down when the 
line joining a point and its tangential is taken. The method of (i) was virtually known long before for equations of the form $y^{3}=f_{3}(x)$. In Euler's Algebra (1784) will be found in addition another method of solving $y^{2}=f_{3}(x)$, which may be expressed geometrically as follows. If $\mathrm{P}$ is a rational point on the cubic, the parabola baving 3-pointic contact at $\mathbf{P}$ of the form $y=a+b x+c x^{2}$, cuts the cubic again in a rational point.

The geometrical method can be extended to other cases which were most probsbly familiar to Lucas, but the extension is more apparent than real, and nuany receive their explanation from the residual theory of Sylvester for cubics.

These usually depend upon the fairly obvious theorem that if two curves of degrees $m$ and $n$ with rational coefficients cut in $m n-1$ rational points, the remaining point of intersection is also a rational point. Particular csses arise for contacts of different orders.

Thus if a conic has 5-pointic contact with a cubic at a rational point $P$, the remaining point of intersection is a rational point; similarly for a rational conic with 3-pointic contact at $P$ and 2-pointic contact at $P^{\prime}$, say, where $P$ and $P^{\prime}$ are rational points.

\$6. We proceed to apply these methods to the cubic (3), viz.,

$$
A y^{2}=x\left(1-x^{2}\right) \text {. }
$$

There are three obvious rational points :- $(0,0) ;(1,0) ;(-1,0)$; but neither method of Cauchy's when applied to these gives any fresh solution. What is more, there can be no further rational points on the cubic if $A$ is a square number, for it is a theorem as old as Fermat that the area $A$ of a rational-sided right-angled triangle can not be a square (Legendre, Theorie des Nombres, Vol. II.).

Let us, however, take any particular triangle and obtain a suitable value for $A$. To this triangle will correspond a perfectly definite rational solution $(\xi, \eta)$ on the cubic $A y^{2}=x\left(1-x^{2}\right)$; for if $\alpha, \beta, \gamma$ are the sides and hypotenuse, then

$$
2 \xi / \eta=\alpha ; \quad\left(1-\xi^{2}\right) / \eta=\beta ; \quad\left(1+\xi^{2}\right) / \eta=\gamma .
$$

Hence $\left(1-\xi^{2}\right) /\left(1+\xi^{2}\right)$ is rational; and $\xi /\left(1-\xi^{2}\right)$ is rational. Hence $\xi^{2}, \xi, \eta$ are rational.

We may therefore start with this rational point as basis. 
\$7. Tangential Method. The tangent at $(\xi, \eta)$ tc

$$
\text { is } \begin{aligned}
A y^{2} & =x\left(1-x^{2}\right) \\
y-\eta & =m(x-\xi)
\end{aligned}
$$

where $m=\left(1-3 \xi^{2}\right) / 2 \mathrm{~A} \eta$. The $x$-eliminant is $x^{3}+\mathrm{A} m^{2} x^{2}+\ldots=0$. Hence if $\left(\xi_{1}, \eta_{1}\right)$ be the tangential of $(\xi, \eta)$ we obtain

$$
\begin{gathered}
2 \xi+\xi_{1}=-A m^{2} \\
\therefore \xi_{1}=-A m^{2}-2 \xi=-\left(\xi^{2}+1\right)^{2} / 4 \xi\left(1-\xi^{2}\right) .
\end{gathered}
$$

Or, if $\alpha, \beta, \gamma$ are the sides and hypotenuse of the first triangle

$$
\xi_{1}=-\gamma^{2} / 2 \alpha \beta=-\left(a^{2}+\beta^{2}\right) / 2 \alpha \beta=-\left(a^{2}+\beta^{2}\right) / 4 A \text {. }
$$

It is unnecessary to calculate $\eta_{1}$, nor need any attention be paid to the sign of $\xi_{1}$. The sides of the new triangle are proportional to $2 \xi_{1}, 1-\xi_{1}^{2}$, and are given by

$$
\lambda\left(2 \gamma^{2} / 2 \alpha \beta\right), \lambda\left(-1+\gamma^{4} / 4 \alpha^{2} \beta^{2}\right) .
$$

Their product is $\alpha \beta$, hence

$$
\lambda^{2} \gamma^{2}\left(\gamma^{4}-4 a^{2} \beta^{2}\right) / 4 a^{3} \beta^{3}=\alpha \beta,
$$

so that

$$
\lambda=2 \alpha^{2} \beta^{2} /\left(a^{2}-\beta^{2}\right) \gamma \text {, if } \alpha>\beta \text {. }
$$

The new triangle therefore has for sides

$$
2 \alpha \beta \gamma /\left(\alpha^{2}-\beta^{2}\right) ;\left(\alpha^{2}-\beta^{2}\right) / 2 \gamma,
$$

and the hypotenuse is $\left(\alpha^{4}+6 a^{2} \beta^{2}+\beta^{4}\right) / 2 \gamma\left(\alpha^{2}-\beta^{2}\right)$.

88. To this analytical result corresponds a simple geometrical construction for the new triangle.

Let $\triangle B C$ be the original triangle. Let $M$ be the middle point of the hypotenuse $A B$, and draw $C D$ perpendicular to $A B$. Then $2 \gamma, M D=\alpha^{2}-\beta^{2}$. Hence one side of the new triangle is the segment MD. This is easily verified directly.

§9. But is a new triangle found? For in an ordinary rightangled triangle it is possible for MD to be equal to a side. Can $a$ or $\beta$ equal $\left(\alpha^{2}-\beta^{2}\right) / 2 \gamma$ when $\gamma^{2}=\alpha^{2}+\beta^{2}$ and all the quantities are rational 9 Let $\xi=\alpha / \gamma, \eta=\beta / \gamma$. Can $\alpha / \gamma$ or $\beta / \gamma=\left(a^{2}-\beta^{2}\right) / 2 \gamma^{3}$ ?

Can

$$
\begin{aligned}
& 2 \xi=\xi^{2}-\eta^{2} ; \xi^{2}+\eta^{2}=1 ; \quad-\quad-\quad \text { - } \\
& 2 \eta=\xi^{2}-\eta^{2} ; \xi^{2}+\eta^{2}=1 \text { ? }
\end{aligned}
$$

The solutions are irrational, hence the new triangle obtained is always distinct from the first.

It naturally follows that the points of inflexion on the curve (3) can not be rational points. 
If we denote the three rational quantities thus found as $a^{\prime}, \beta^{\prime}, \gamma^{\prime}$, the next triangle would have one side equal to

$$
\begin{gathered}
\pm 2 a^{\prime} \beta^{\prime} \gamma^{\prime} /\left(\alpha^{\prime 2}-\beta^{\prime 2}\right), \\
\text { or } \pm 4 a \beta \gamma\left(\alpha^{2}-\beta^{2}\right)\left(\alpha^{4}+6 a^{2} \beta^{2}+\beta^{4}\right) /\left\{16 \alpha^{2} \beta^{2} \gamma^{4}-\left(\alpha^{2}-\beta^{2}\right)^{4}\right\}= \pm D \text {, say. }
\end{gathered}
$$

This can not equal $\alpha^{\prime}$ or $\beta^{\prime}$. Can it be equal to $\alpha$ or $\beta$ ?

The relation $a= \pm D$ leads to the equations

$$
16 \xi^{2} \eta^{2}-\left(\xi^{2}-\eta^{2}\right)^{4}= \pm 4 \eta\left(\xi^{2}-\eta^{2}\right)\left(\xi^{4}+6 \xi^{2} \eta^{2}+\eta^{4}\right) ; \xi^{2}+\eta^{2}=1 \text {. }
$$

Eliminate $\xi$, and $\eta$ is a root of the equation

$$
16 \eta^{8}-32 \eta^{6}+40 \eta^{4}-24 \eta^{2}+1 \pm 4 \eta\left(1+2 \eta^{2}-12 \eta^{4}+8 \eta^{6}\right)=0 .
$$

Write $y / 2$ for $\eta$ when we deduce an equation in $y$,

$$
y^{8}+\ldots+16=0
$$

where the coefficients are integers. Any rational solution of this equation must be an integer, and can therefore only be $\pm 1 ; \pm 2 ; \pm 4 ; \pm 8 ; \pm 16$; so that any rational root in $\eta$ must be $\pm 1 / 2 ; \pm 1$; etc. But $\xi= \pm \sqrt{1-\eta^{2}}$, and can be rational for only one of these values, viz., when $\eta= \pm 1$. But $\xi$ would then be zero, which is impossible from the nature of the problem. On writing $\beta= \pm D$, the same equation is obtained in $\xi$ and similar conclusions are deduced. The final conclusion therefore is that the three triangles thus found are distinct equivalent and rational-sided right-angled triangles, and Carroll's problem is therefore solved. If the sides are to be integers, a suitable numerical factor can always be introduced. Owing to the restriction that the solutions must always be rational, it is very probable that the series could be indefinitely incressed, but it is quite easy to construct a cubic such that even the third tangential of a point on it coincides with the point itself for certain positions on the cubic.

$\$ 10$. The application of the chord residue method (ii) of Cauchy leads to some interesting conclusions.

It is also noteworthy that in this case the solutions obtained by the tangential method may be found by the second method.

More generally, if three points $\mathrm{A}, \mathrm{B}, \mathrm{C}$ on a cubic are known no one of which is a tangential of another, the tangential of $A$, say, may be found as follows: 
Lot $A B$ and $A C$ cut the curve again in $B^{\prime}$ and $C^{\prime}$. Then by Theorem I. we have the collinear points given by
A B B
$A \quad C^{\prime}$
T D D'

where $\mathrm{D}$ and $\mathrm{D}^{\prime}$ are the points in which $\mathrm{BC}$ and $\mathrm{B}^{\prime} \mathrm{C}^{\prime}$ again cut the cubic, and $T$ is the tangential of $A$.

Now the cubic

$$
\mathrm{A} y^{2}=x\left(1-x^{2}\right)
$$

possesses three rational points $(0,0) ;(-1,0) ;(1,0)$ which may be denoted by $\mathrm{O}, \mathrm{O}_{1}, \mathrm{O}_{2}$. If therefore a rational point $P_{1}$ distinct from these is known, the tangential $Q_{1}$ of $P_{1}$ can be found by the residual method. It is remarkable, however, that although new rational points on the curve are found by joining $P_{1}$ to the points " $O$," no new solution of the problem is thereby directly obtained.

Let $(\xi, \eta)$ be the coordinates of $\mathrm{P}_{1}$ and let $\mathrm{P}_{1} \mathrm{O}, \mathrm{P}_{1} \mathrm{O}_{2}, \mathrm{P}_{1} \mathrm{O}_{1}$ cut the curve again in $P_{2}, P_{3}, P_{4}$. It is easily shown that these points are

$\left(-1 / \xi,-\eta / \xi^{2}\right) ;\left((\xi+1) /(\xi-1), 2 \eta /(\xi-1)^{2}\right) ;\left((1-\xi) /(1+\xi), 2 \eta /(\xi+1)^{2}\right)$.

Consider the ratio of the sides of the triangle corresponding to $P_{1}$. It is given by $2 \xi /\left(1-\xi^{2}\right)$.

Now the solutions in $x$ of the equation and those of

$$
2 x /\left(1-x^{2}\right)= \pm 2 \xi /\left(1-\xi^{2}\right) \text { are } \pm \xi, \pm 1 / \xi
$$

$$
2 x /\left(1-x^{2}\right)= \pm\left(1-\xi^{2}\right) / 2 \xi \text { are } \pm(1+\xi) /(1-\xi) \text { and } \pm(1-\xi) /(1+\xi) \text {. }
$$

The ratio of the sides is therefore unaltered by selecting $P_{2}, P_{3}$, or $P_{4}$, and as the area is unaltered no new triangles are formed.

$\$ 11$. There can likewise be no new solutions found by joining $P_{2}, P_{8}, P_{4}$ to the neutral points $O$, etc., but the number of points found in this way is limited. If $P_{1}^{\prime}, P_{2}^{\prime}$, etc., are the images of $P_{1}, P_{2}$, etc., in the $x$-axis, the following table contains only eight distinct points $\mathbf{P}$.
(1) $\mathrm{P}_{1} \mathrm{OP}_{2} ; \mathrm{P}_{2} \mathrm{O}_{1} \mathrm{P}_{4} ; \mathrm{P}_{2} \mathrm{O}_{2} \mathrm{P}_{3}$
(2) $\mathrm{P}_{2} \mathrm{OP}_{1} ; \mathrm{P}_{2} \mathrm{O}_{1} \mathrm{P}_{3}^{\prime} ; \mathrm{P}_{2} \mathrm{O}_{2} \mathrm{P}_{4}^{\prime}$
(3) $\mathrm{P}_{3} \mathrm{OP}_{4}^{\prime} ; \mathrm{P}_{3} \mathrm{O}_{1} \mathrm{P}_{2}^{\prime} ; \mathrm{P}_{3} \mathrm{O}_{2} \mathrm{P}_{1}$
(4) $\mathrm{P}_{4} \mathrm{OP}_{3}^{\prime} ; \mathrm{P}_{4} \mathrm{O}_{1} \mathrm{P}_{1} ; \mathrm{P}_{4} \mathrm{O}_{2} \mathrm{P}_{2}^{\prime}$ 
with four similar rows formed by interchanging dashed and undashed letters $\mathrm{P}$, - a transformation following from the symmetry of the cubic.

Theorem I. readily establishes these, or they may be verified analytically. Thus, to establish $\mathrm{P}_{2} \mathrm{O}_{1} \mathrm{P}_{3}^{\prime}$, we have the systom

$$
\begin{array}{lll}
\mathrm{P}_{2} & \mathrm{O} & \mathrm{P}_{1} \\
\mathrm{O}_{1} & \mathrm{O} & \mathrm{O}_{2} \\
& \infty & \mathrm{P}_{3},
\end{array}
$$

where $\infty$ denotes the point at infinity on the $y$-axis where the tangent at $O$ again cuts the curve. But the line joining $P_{3}$ to this point is perpendicular to the $x$-axis, and therefore passes through $P_{s}^{\prime}$. In this way groups of eight points are obtained. We proceed to examine a group of these in detail, and to apply method (ii) to them.

$\$ 12$. If $Q_{1}$ is the tangential of $P_{1}$, it is also the tangential of $P_{2}^{\prime}, P_{3}^{\prime}, P_{4}^{\prime}$; and $Q_{1}^{\prime}$ is the tangential of $P_{1}^{\prime}, P_{2}, P_{3}, P_{4}$.

For by $I$. we have the array

$$
\begin{array}{lll}
P_{1} & O & P_{2} \\
P_{1} & P_{3} & O_{2} \\
Q_{1} & P_{4}^{\prime} & P_{4}^{\prime}
\end{array}
$$

which proves that $P_{4}^{\prime}$ has $Q_{1}$ for tangential.

Cor. If $Q$ is the tangential of a rational point, the four tangents that can be drawn from it to touch the curve elsewhere are rational. Or, if one of the tangents from a point on the curve is rational, so are the other three, and each meets the curve in rational points.

$\S 13$. The chord residue method will, in fact, be found less fruitful in new results then might have been expected.

Consider the chords

$$
\mathbf{P}_{1} \mathbf{P}_{2}^{\prime} ; \mathbf{P}_{\mathbf{1}} \mathbf{P}_{3}^{\prime} ; \mathbf{P}_{\mathbf{1}} \mathbf{P}_{\mathbf{4}}^{\prime} \text {. }
$$

Let $Q_{1}$ when joined to the neutral points $O, O_{1}, O_{2}$ give rise to the group $\left(Q_{1} \ldots \ldots Q_{t}^{\prime}\right)$.

We then find the following triads

$$
P_{1} P_{2}^{\prime} Q_{2}^{\prime}, P_{1} P_{3}^{\prime} Q_{3}^{\prime}, P_{1} P_{4}^{\prime} Q_{4}^{\prime} \text {. }
$$

To establish the first of these we have

$$
\begin{array}{llllllll}
\mathbf{P}_{1} & \mathbf{P}_{2}^{\prime} & & & & & & \\
\mathbf{P}_{1} & \mathbf{P}_{3} & \mathbf{O}_{2} & & & & \\
\mathbf{Q}_{1} & \mathbf{O}_{1} & \mathbf{Q}_{1} & \therefore & \mathbf{P}_{1} & \mathbf{P}_{3}^{\prime} & \mathbf{Q}_{2}^{\prime} .
\end{array}
$$


Similarly from $\mathrm{P}_{2}$ we obtain

and from $P_{3}$

$$
\mathrm{P}_{2} \mathrm{P}_{3} \mathrm{Q}_{4} ; \quad \mathrm{P}_{2} \mathrm{P}_{4} \mathrm{Q}_{3} ; \quad \mathbf{P}_{2} \mathrm{P}_{1}^{\prime} \mathrm{Q}_{2} ;
$$

The possible new triads for $P_{1}^{\prime}$, etc., may be obtained by symmetry.

There results no new triangle distinct from that for $Q_{1}$ by joining points $\mathbf{P}$.

\$14. Let $R_{1}$ be the second tangential of $P_{1}$ and the first tangential of $Q_{1}$. The preceding will now apply to the group of points $Q$. Consider the new points to be found by joining a $P$ and a $Q$.

Let $Q_{1} P_{2}$ cut the cubic again in $X_{2}$, and let $X_{2} O$ cut again in $X_{1}$. Form the octad of points corresponding to $X_{1}$.

The lines joining $Q_{1}$ to $P_{1}, P_{2}^{\prime}, P_{3}^{\prime}, P_{4}^{\prime}$ lead to no new point, and we therefore should discuss $Q_{1} P_{2}, Q_{1} P_{3}, Q_{1} P_{4}, Q_{1} P_{1}$.

It will be shown presently that these lead to $X_{2} X_{3} X_{4} X_{1}^{\prime}$, i.e., to a system of points possessing a common tangential.

\$15. Use Theorem I. for the array in which the first row corresponds to a tangent from $Q_{1}$, the second row to the line $P_{2} P_{1} Q_{2}$, and there results

$$
\mathbf{Q}_{2} \mathrm{P}_{1} \mathrm{X}_{2}^{\prime} ; \quad \mathrm{Q}_{2} \mathrm{P}_{2}^{\prime} \mathrm{X}_{1} ; \quad \mathbf{Q}_{2} \mathrm{P}_{3}^{\prime} \mathrm{X}_{4}^{\prime} ; \quad \mathbf{Q}_{3} \mathbf{P}_{4}^{\prime} \mathrm{X}_{\mathrm{s}}^{\prime} \text {. }
$$

Replace the second row by $P_{2} P_{4} Q_{3}$, and we find

$$
Q_{3} P_{1} X_{3}^{\prime} ; \quad Q_{3} P_{2}^{\prime} X_{4}^{\prime} ; Q_{3} P_{3}^{\prime} X_{1} ; Q_{3} P_{4}^{\prime} X_{2}^{\prime} \text {. }
$$

Take $P_{9} P_{3} Q_{4}$ for the second row of $I$., and we find

$$
\mathbf{Q}_{1} \mathbf{P}_{1} \mathbf{X}_{4}^{\prime} ; \quad \mathbf{Q}_{4} \mathbf{P}_{2}^{\prime} \mathbf{X}_{3}^{\prime} ; \quad \mathbf{Q}_{4} \mathbf{P}_{3}^{\prime} \mathbf{X}_{2}^{\prime} ; \mathbf{Q}_{4} \mathbf{P}_{4}^{\prime} \mathbf{X}_{1} \text {. }
$$

In these we may interchange dashed and undashed letters. Hence

i.e., $\mathrm{Q}_{1} \mathrm{P}_{3} \mathrm{X}_{3}$.

$$
\begin{array}{lll}
\mathbf{Q}_{1} & \mathbf{P}_{3}^{\prime} & \mathbf{P}_{2}^{\prime} \\
\mathbf{P}_{3} & \mathbf{O} & \mathrm{P}_{1} \\
\mathrm{X}_{3} & \mathrm{P}_{4} & \mathbf{Q}_{2}^{\prime} .
\end{array}
$$

We also obtain the arrays

$$
\begin{array}{lllllll}
Q_{1} & P_{2}^{\prime} & P_{2}^{\prime} & & O & O_{1} & O_{2} \\
P_{1} & O_{1} & P_{1} & \text { and } & Q_{2} & P_{4}^{\prime} & X_{3}^{\prime} \\
X_{4} & P_{3} & Q_{2}^{\prime} & & Q_{1} & P_{1}^{\prime} & X_{1}^{\prime},
\end{array}
$$

hence $Q_{1} P_{4} X_{6} ; Q_{1} P_{1}^{\prime} X_{1}^{\prime}$.

The other joins of $P$ 's and Q's are already accounted for. 
$\S 16$. The tedious process of the preceding paragraph may be somewhat curtailed by the following considerations along with a proper arrangement of the points.

The rational points so far obtained are $\left(\mathrm{OO}_{1} \mathrm{O}_{2} \infty\right) ;\left(\mathrm{P}_{1} \mathrm{P}_{2}^{\prime} \mathrm{P}_{3}^{\prime} \mathrm{P}_{4}^{\prime}\right) ;\left(\mathrm{P}_{1}^{\prime} \mathrm{P}_{2} \mathrm{P}_{3} \mathrm{P}_{4}\right) ;\left(\mathrm{Q}_{1} \mathrm{Q}_{2}^{\prime} \mathrm{Q}_{3}^{\prime} \mathrm{Q}_{4}^{\prime}\right) ;\left(\mathrm{Q}_{2}^{\prime} \mathrm{Q}_{2} \mathrm{Q}_{3} \mathrm{Q}_{4}\right)$;

$$
\left(\mathrm{X}_{1} \mathrm{X}_{2}^{\prime} \mathbf{X}_{3}^{\prime} \mathbf{X}_{4}^{\prime}\right) ;\left(\mathbf{X}_{1}^{\prime} \mathbf{X}_{2} \mathbf{X}_{3} \mathbf{X}_{4}\right) \text {; }
$$

in which members possessing a common tangential are grouped. It will be seen that if any member of one group of four points is joined to another group of four points, the same group of four points bas been obtained. This follows from the following more general theorem :-

If any member of a group of four points possessing a common tangential is joined to a similar group of four points, the same four points of intersection of joins with cubic are obtained and the latter possess a common tangential.

Let $A, B, C, D$ be four points on a cubic having the common tangential $T$. Let $P$ be any other point, and let $P A$ cut again in $A_{1}$. Let the tangential of $P$ be $Q$ and of $A$ be $T$. Then by Theorem $I$.

$\therefore \mathrm{QTT}_{1}$ are in a line.

$$
\begin{array}{lll}
\mathbf{P} & \mathbf{P} & \mathbf{Q} \\
\mathrm{A} & \mathrm{A} & \mathrm{T} \\
\mathrm{A}_{1} & \mathrm{~A}_{1} & \mathrm{~T}_{1} ;
\end{array}
$$

But $Q$ is fixed and $T$ is fixed ; therefore $T_{1}$ is a fixed point, and the same point $T_{1}$ is the tangential of $B_{1}, C_{1}, D_{1}$. Also the point $Q$ is the same for the four points $P$ possessing $Q$ as a common tangential. Hence the theorem follows.

$\$ 17$. It will be observed that the points $\mathrm{OO}_{1} \mathrm{O}_{2} \infty$ form such a system of four points, their tangential being the inflexional point at infinity. It may also be noted that the methods of proof hitherto employed would apply to any non-singular cubic, only for images of points the corresponding harmonic conjugates with respect to a point of inflexion require to be taken. So far as our problem is concerned no distinction is made among points possessing a common tangential.

The following notation may therefore be used with the object of finding fresh solutions.

Let $P$ denote indifferently any one of the four points $\mathbf{P}_{2}, \mathbf{P}_{2}^{\prime}, \mathbf{P}_{3}^{\prime}, \mathbf{P}_{4}^{\prime}$; and $\overrightarrow{\mathbf{P}}$ its image (or harmonic conjugate). 
Let the successive tangentials of $P$ be $Q, R, S$; and $\therefore$ of $\bar{P}$ be $\overline{\mathbf{Q}}, \overline{\mathbf{R}}, \overline{\mathbf{S}}$. Let $\mathbf{P Q}$ cut in $\mathrm{X}, \mathrm{Q} \overline{\mathrm{R}}$ in $\mathrm{Y}, \mathrm{PR}$ in $\mathrm{Z}, \mathrm{PY}$ in $\mathrm{U}, \mathrm{R} \overline{\mathrm{S}}$ in $\zeta$.

\$18. The following table of collinear points may then be easily constructed.

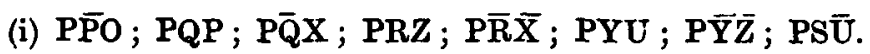

(ii) $\overline{\mathrm{PPO}}$; $\overline{\mathrm{P}} \overline{\mathrm{Q}} \overline{\mathrm{P}}$; etc.

(iii) $Q \bar{Q} \mathrm{Q}$; $Q R Q ; Q \overline{R Y} ; \mathbf{Q X \overline { Z }} ; \mathbf{Q} \overline{\mathbf{X}} \bar{P} ; \mathbf{Q} \bar{Y} \overline{\mathrm{S}} ; \mathrm{QZ} \overline{\mathrm{U}}$.

(iv) $\overline{\mathbf{Q} Q O} ; \overline{\mathrm{Q}} \overline{\mathbf{Q}} \mathbf{Q}$; etc.

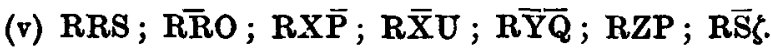

(vi) $\overline{\mathbf{R}} \overline{\mathrm{RS}}$; etc.

(vii) $\mathrm{XXY} ; \mathbf{X} \overline{\mathbf{X}} \mathbf{X} ; \mathbf{X Z} \overline{\mathbf{S}} ; \mathbf{X} \overline{\mathbf{Z}} \mathbf{Q}$.

(viii) $\bar{X} \bar{X} \bar{Y}$; etc.

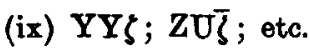

All the possible solutions thus obtained for Carroll's problem would not be greater than nine in number, as corresponding to

$$
\mathrm{P}, \mathrm{Q}, \mathbf{R}, \mathrm{S}, \mathrm{X}, \mathbf{Y}, \mathrm{Z}, \zeta, \mathrm{U} \text {. }
$$
of $\mathrm{X}$.

$\$ 19$. It will be observed that $Y$ and $\zeta$ are successive tangentials

For

$$
\begin{array}{rrrrr} 
& \mathbf{P} & \mathbf{P} & \mathbf{Q} \\
& \overline{\mathbf{Q}} & \overline{\mathbf{Q}} & \overline{\mathbf{R}} \\
\therefore & \mathbf{X} & \mathbf{X} & \mathbf{Y} .
\end{array}
$$

In the construction of the preceding table $(\S 18)$ the following theorems are also useful.

Let $P$ and $A$ be any two points on the cubic, and let $P A B, P \bar{A} C$ be collinear points on the cubic. Then $B C$ passes through a point $\bar{Q}$.

For

$$
\begin{array}{rrrr} 
& \mathbf{P} & \mathbf{P} & \mathbf{Q} \\
\mathbf{A} & \overline{\mathbf{A}} & \mathbf{O} \\
\therefore & \mathbf{B} & \mathbf{C} & \overline{\mathbf{Q}} .
\end{array}
$$

Also the residual points corresponding to $A C$ and $\bar{A} B$ are in a line with $\mathbf{Q}$.

For

$$
\begin{array}{rrrr} 
& \mathbf{P} & \mathbf{A} & \mathbf{B} \\
& \mathbf{P} & \mathbf{C} & \overline{\mathbf{A}} \\
\therefore & \mathbf{Q} & . &
\end{array}
$$


$\$ 20$. It might readily be imagined that a convenient algorithm for finding new solutions would be found as follows.

Let $\mathrm{P}_{1} \mathrm{AB}$ be three rational points on the cubic and use the residual method to determine the points $P_{2}, P_{3} \ldots$ from the table

$$
\begin{array}{lll}
\mathrm{P}_{1} & \mathrm{~A} & \mathrm{P}_{2} \\
\mathrm{P}_{2} & \mathrm{~B} & \mathrm{P}_{3} \\
\mathrm{P}_{3} & \mathrm{~A} & \mathrm{P}_{4} \\
\mathrm{P}_{4} & \mathrm{~A} & \mathrm{P}_{5} \text {, etc. }
\end{array}
$$

Unfortunately the very first case one takes breaks down rapidly. Take the points $\mathrm{P}_{1}, \mathrm{O}_{1}, \mathrm{O}$.

We find

$$
\begin{array}{lll}
P_{1} & O_{1} & P_{4} \\
P_{4} & O & P_{3}^{\prime} \\
P_{3}^{\prime} & O_{1} & P_{2} \\
P_{2} & O & P_{1}
\end{array}
$$

and we only obtain the four points $P_{1} P_{4} P_{3}{ }^{\prime} P_{2}$.

$\$ 21$. This is a particular case of the following theorem.

If $A$ and $B$ have a common tangential and we start with $P_{1}$ any point on the cubic, we obtain

$$
\begin{array}{lll}
\mathbf{P}_{1} & \mathrm{~A} & \mathbf{P}_{2} \\
\mathbf{P}_{2} & \mathrm{~B} & \mathbf{P}_{3} \\
\mathbf{P}_{3} & \mathrm{~A} & \mathbf{P}_{4} \\
\mathbf{P}_{4} & \mathrm{~B} & \mathbf{P}_{1}
\end{array}
$$

For let the common tangential of $A$ and $B$ be $T$, and assume the first three rows furnishing $P_{2} P_{3} P_{4}$ to prove $P_{4} B P_{1}$.

We find

$$
\begin{array}{lll}
\mathrm{P}_{1} & \text { A } & \mathrm{P}_{2} \\
\mathrm{P}_{3} & \mathrm{~A} & \mathrm{P}_{4}
\end{array}
$$

$\therefore R$ T $S$, say, where $R$ and $S$ are the residuals of $P_{1} P_{3}$ and $P_{2} P_{4}$ respectively.

Also

$$
\begin{array}{lll}
\mathrm{P}_{2} & \mathrm{~B} & \mathrm{P}_{3} \\
\mathrm{P}_{4} & \mathrm{~B} & ? \\
\mathrm{~S} & \mathrm{~T} & \mathrm{R} .
\end{array}
$$

$\therefore P_{3} R$ and $P_{4} B$ cut in the same point $P_{1}$.

This theorem is Prop. XVI. of Maclaurin's Treatise on the General Properties of Geometrical Lines, and contains the germ of what are generally termed Steiner's Polygons, viz. :- 
"Let $A$ and $B$ be two points on a cubic, $P_{1}$ any point such that the system

$$
\begin{array}{lll}
\mathrm{P}_{1} & \mathrm{~A} & \mathrm{P}_{2} \\
\mathrm{P}_{2} & \mathrm{~B} & \mathrm{P}_{3} \text {, etc., }
\end{array}
$$

begins to repeat after $P_{2 n}$, then this will happen for any other point $P$ on the cubic." (vide Schröter $l$. c.)

The conditions under which this happens are furnished by the same authority.

$\$ 22$. It might be expected that if we had three points $A, B, C$ and a point $P$, then by forming the system

$$
\begin{array}{lll}
P_{1} & A & P_{2} \\
P_{2} & B & P_{y}, \text { etc., }
\end{array}
$$

we should obtain better results.

But if $\mathrm{ABC}$ are to be repeated cyclically, only five new points are obtained, and the points repeat after $P_{6}$. In this case $A, B, C$ may be any three points whatsoever on the curve.

Form the table
$\begin{array}{lll}A & P_{1} & P_{2}\end{array}$
\begin{tabular}{llll} 
B & $\mathrm{P}_{2}$ & $\mathrm{P}_{3}$ \\
\hline
\end{tabular}
$\begin{array}{lll}\mathrm{C} & \mathrm{P}_{3} & \mathbf{P}_{4}\end{array}$
A $\quad P_{4} \quad P_{5}$
$\begin{array}{llll}B & P_{5} & P_{6}\end{array}$
C $P_{6}$ ?.

From these we deduce the array

$$
\begin{array}{llllllll}
\mathrm{P}_{1} & \mathrm{~A} & \mathrm{P}_{2} & & & & & \\
\mathrm{P}_{6} & \mathrm{P}_{5} & \mathrm{~B} & & & & & \\
\mathrm{C} & \mathbf{P}_{4} & \mathrm{P}_{3} & & \therefore & \mathrm{C} & \mathbf{P}_{6} & \mathbf{P}_{1} ;
\end{array}
$$

i.e., we come back to the point $P_{1}$ from which we started.

This again is the first of a series of theorems.

"If $n$ is an odd number, $A_{1}, A_{3}, \ldots A_{n}, n$ points on a cubic, $P$ any other point on the cubic, the polygon formed as in the preceding closes up at $P_{2 n}$ after cyclical use of the points $A$ twice." (Schröter l. c.) 